\title{
Inhibition of RANKL-induced osteoclast differentiation through the downregulation of c-Fos and NFATc1 by Eremochloa ophiuroides (centipedegrass) extract
}

\author{
BO-YUN CHOI ${ }^{1}$, CHUL-HONG PARK ${ }^{1,2}$, YUN HEE NA ${ }^{1,3}$, HYOUNG-WOO BAI $^{1}$, \\ JAE-YOUNG $\mathrm{CHO}^{4}$ and BYUNG YEOUP $\mathrm{CHUNG}^{1}$
}

\author{
${ }^{1}$ Research Division for Biotechnology, Advanced Radiation Technology Institute, \\ Korea Atomic Energy Research Institute, Jeongeup, North Jeolla 580-185; ${ }^{2}$ School of Biological Sciences and Biotechnology; \\ ${ }^{3}$ Interdisciplinary Graduate Program in Molecular Medicine, Chonnam National University, Gwangju 500-757; \\ ${ }^{4}$ Department of Applied Life Sciences, Chonbuk National University, Jeonju, North Jeolla 561-756, Republic of Korea
}

Received March 2, 2015; Accepted December 31, 2015

DOI: $10.3892 / \mathrm{mmr} .2016 .5015$

\begin{abstract}
Osteoclasts, derived from hematopoietic stem cells, are specialized macrophages and have a homeostatic role in skeletal modeling and remodeling with bone-forming osteoblasts. However, excessive osteoclast activity induces bone diseases, including osteoporosis, periodontitis and rheumatoid arthritis. Natural substances have received attention as therapeutic drugs in human diseases. In the current study, cells isolated from mouse bone marrow, and a mouse model, were used to determine the effect of centipedegrass extract (CGE) on osteoclasts. Multiple concentrations of CGE were administered to bone marrow cells for 24-72 hours and, for the in vivo study, mice were treated with CGE for 8 days. The effects of CGE on transcription and translation of osteoclast-associated molecules were then determined using reverse transcription-polymerase chain reaction and immunoblotting, respectively. In the present study it was shown that CGE extracted from Eremochloa ophiuroides (centipedegrass) inhibited receptor activator of nuclear factor $\kappa$-B ligand (RANKL)-mediated osteoclast differentiation in bone marrow macrophages, without cytotoxicity, in a dose-dependent manner. CGE decreased the expression levels of osteoclast-specific genes, including matrix metalloproteinase-9, osteoclast-associated immunoglobulin-like receptor and cathepsin $\mathrm{K}$, however, CGE had no inhibitory effect on the expression levels of mitogen-activated protein kinases, nuclear factor- $\kappa \mathrm{B}$ and Akt. Furthermore, the protein
\end{abstract}

Correspondence to: Dr Byung Yeoup Chung, Research Division for Biotechnology, Advanced Radiation Technology Institute, Korea Atomic Energy Research Institute, 1266 Sinjeong-dong, Jeongeup, North Jeolla 580-185, Republic of Korea

E-mail: bychung@kaeri.re.kr

Key words: osteoclast, osteoclastogenesis, Eremochloa ophiuroides, centipedegrass, receptor activator of nuclear factor $\kappa$-B ligand, bone and RNA levels of RANKL-induced c-Fos and nuclear factor of activated T-cell cytoplasmic 1 were suppressed by CGE. These results indicated that CGE may serve as a useful drug in the prevention of bone loss.

\section{Introduction}

Bone homeostasis is a dynamic process, which maintains bone skeletal mass and quality, and protects organs and hematopoiesis by bone remodeling throughout life $(1,2)$. Bone remodeling is a physiological process that involves a functional balance between osteoclast-regulated bone resorption and osteoblast-induced bone formation $(3,4)$. However, an imbalance in bone remodeling, induced by various diseases, aging, smoking, increasing inflammation factors or estrogen deficiency following menstruation, leads to adult skeletal defects, including osteoporosis, rheumatoid arthritis, Paget's disease and osteolysis associated with periodontal disease (5). These diseases are characterized by the excessive activity of osteoclasts (5).

Osteoclasts are bone-resorbing multinuclear cells, which originate from hematopoietic stem cells of the monocyte/macrophage lineage (6). The differentiation of osteoclasts is regulated by the two cytokines, receptor activator of nuclear factor (NF) $\kappa-\mathrm{B}$ ligand (RANKL) and macrophage colony-stimulating factor (M-CSF) (7). M-CSF provides survival signals and induces the expression of the RANK receptor in osteoclast and precursor cells (1). RANKL is expressed in osteoblasts and binds to the RANK receptor expressed in osteoclast precursor cells $(1,8)$. This binding leads to the activation of key signaling pathways in osteoclast differentiation through activated osteoclasia and the regulation of factors assisting bone resorption (7). RANKL is a member of the tumor necrosis factor (TNF) superfamily, which is expressed in osteoblasts $(4,9)$. Binding of RANKL to its receptor, RANK, on osteoclast precursors results in the recruitment of TNF receptor-associated factor (TRAF) family proteins, including TRAF6 (10). TRAF6 induces not only mitogen-activated protein kinases (MAPKs), but also activates $\mathrm{NF}-\kappa \mathrm{B}$ (2). This signaling subsequently activates the c-Fos 
transcription factor and nuclear factor of activated $\mathrm{T}$ cell cytoplasmic 1 (NFATc1) $(10,11)$. These transcription factors regulate the expression of several osteoclast-specific genes (3).

To identify compounds, which inhibit osteoclast differentiation, the present study used centipedegrass extract (CGE) in a bone marrow culture system. During the process, it was investigated whether osteoclast formation was suppressed by Eremochloa ophiuroides CGE. E.ophiuroides is native to China and Southeast Asia, and has become one of the most popular lawn grasses in South America $(12,13)$. In a previous study, maysin was identified as a component of E.ophiuroides centipedegrass by liquid chromatography-mass spectrometry (12). CGE also contains several C-glycosyl flavones and phenolic constituents, including luteolin, orientin, isoorientin, rhamnosylisoorientin, derhamnoslymaysin and luteoin-6-C-boivinopyranose $(12,14)$. A previous study reported that the methanolic extracts from the leaves of E. ophiuroides centipedegrass exhibit anticancer and anti-adipogenic activities in several cell types $(12,15)$.

However, there are no reports on the inhibitory effects of CGE on osteoclastogenesis. In the present study, the anti-osteoclastogenic effects and underlying signaling pathway of CGE in RANKL-stimulated primary precursor cells without cytotoxity were investigated. The results demonstrated for the first time, to the best of our knowledge, that CGE significantly suppresses RANKL-induced osteoclast differentiation by modulating osteoclast-specific genes, transcription factors and signaling molecules (16). Thus, these results provided evidence that CGE may represent an anti-osteoclastogenic agent for use in the treatment of bone loss diseases.

\section{Materials and methods}

Materials and antibodies. Cell culture medium and fetal bovine serum (FBS) were obtained from Invitrogen (Thermo Fisher Scientific, Inc., Waltham, MA, USA). RANKL and M-CSF were purchased from PeproTech (Rocky Hill, NJ, USA). The Leukocyte Acid Phosphatase Assay kit and 3-(4,5-dimethylthiazol-2-yl)-2,5-diphenyltetrazolium bromide (MTT) were purchased from Sigma-Aldrich (St. Louis, MO, USA).

Preparation of the CGE. The dried leaves of E. ophiuroides (centipedegrass; $5 \mathrm{~kg}$ ) were ground in a Wiley mill (Weiber, India) and passed through a $420-\mu \mathrm{m}$ sieve. The final ground sample $(1 \mathrm{~kg})$ was extracted three times with 100 liters of $80 \%$ methanol $(\mathrm{MeOH} ; \mathrm{v} / \mathrm{v})$ for $24 \mathrm{~h}$ with constant agitation at an ambient temperature in the dark. The extracts were filtered using No. 2 filter paper (Advantech Japan Co., Ltd., Tokyo, Japan) and concentrated in vacuo. The $\mathrm{MeOH}$ extracts were then fractionated with n-hexane and ethyl acetate (EA)(Daejung Chemicals \& Metals Co., Ltd., Siheung, Korea). The EA extracts were concentrated in vacuo using an EYELA N-1001 rotary evaporator (Tokyo Rikakikai Co., Ltd., Tokyo, Japan) and the dried compounds were dissolved in $\mathrm{MeOH}$. The dissolved $\mathrm{MeOH}$ extracts were diluted in $20 \% \mathrm{MeOH}$ and chromatographed on a Toyopearl HW-40C resin (Tosoh, Tokyo, Japan) column using $70 \% \mathrm{MeOH}$ (elution volume, $700 \mathrm{ml}$ ). The fraction was evaporated and freeze-dried, and the dried extracts were reconstituted in dimethyl sulfoxide (DMSO; final concentration, $1 \mathrm{mg} / \mathrm{ml}$; Sigma-Aldrich) for cell treatment. CGE was analyzed using an HPLC-MS (Agilent 1260 series; Agilent Technologies,
Santa Clara, CA, USA) in a positive ion mode. The CGE was injected $(10 \mu \mathrm{l})$ and chromatographed on a YMC-Pack ODS-A (150x4.6 mm; 1.D. S-5 $\mu 1 ; 12 \mathrm{~nm}$ ) with a linear gradient between buffer A (0.1\% formic acid; Wako Pure Chemical Industries, Ltd., Osaka, Japan) and buffer B (100\% MeOH). The mobile phase was programed as follows: $100 \%$ buffer A at $10 \mathrm{~min}$, $50 \%$ buffer $\mathrm{A}$ at $30 \mathrm{~min}$ and $0 \%$ buffer $\mathrm{A}$ at $60 \mathrm{~min}$. The calibration curves were generated using high performance liquid chromotography (HPLC) with standards, including rhamnosylisoorientin, derhamnoxylmaysin, maysin, eremonetin (all kindly donated by Tae Hoon Kim, Daegu University, South Korea), chlorogenic acid, orientin, isoorientin, luteolin and icariin (used as a standard compound) (all Sigma-Aldrich; data not shown). The flow rate was $0.5 \mathrm{ml} / \mathrm{min}$ and ultraviolet detection was performed at $360 \mathrm{~nm}$ with a 1260 Infinity Diode Array Detector (Agilent Technologies, Santa Clara, CA, USA). The CGE contained predominantly chlorogenic acid, at $\sim 20 \%$ [43.4 $\mu \mathrm{g} / \mathrm{mg}$ dry weight(D.W)], derahnosylmaysin at $\sim 65 \%$ (108.4 $\mu \mathrm{g} / \mathrm{mg}$ D.W), maysin at $\sim 5 \%$ (193.6 $\mu \mathrm{g} / \mathrm{mg}$ D.W), as shown in Fig. 1.

Isolation of bone marrow-derived macrophages (BMMs) and cell culture. A total of 5 male ICR mice (5-week-old; body weight, 30-32 g) were obtained from SLC, Inc. (Kotoh-cho, Japan). The mice were maintained under a 12-h light/dark cycle, with access to food and water ad libitum and allowed to acclimate to their environment for 1 week prior to beginning experiments. To isolate the BMMs, marrow cells were cultured from the femurs and tibias of 5 mice in $\alpha$-minimal essential medium $(\alpha-\mathrm{MEM})$ containing 10\% FBS with $100 \mathrm{U} / \mathrm{ml}$ penicillin and $100 \mu \mathrm{g} / \mathrm{ml}$ streptomysin (Gibco; Thermo Fisher Scientific, Inc.) in the presence of M-CSF (30 ng/ml) at $37^{\circ} \mathrm{C}$ in $5 \% \mathrm{CO}_{2}$. After 3 days, the nonadherent cells were removed by washing with fresh medium, and the adherent cells were used as BMMs. The BMMs were further cultured for 3 days in an osteoclastogenic medium ( $\alpha$-MEM containing $10 \% \mathrm{FBS}$, $30 \mathrm{ng} / \mathrm{ml} \mathrm{M-CSF}$ and $100 \mathrm{ng} / \mathrm{ml}$ RANKL). The cells were then cytochemically stained for tartrate-resistant acid phosphatase (TRAP), an osteoclast marker protein.

TRAP staining. The BMMs were replated and then further cultured in medium containing M-CSF $(30 \mathrm{ng} / \mathrm{ml})$ and RANKL (100 ng/ml) for 3 days. The cells were washed with phosphate-buffered saline (PBS) and fixed $3.7 \%$ formaldehyde (Sigma-Aldrich) for $10 \mathrm{~min}$. Following washing with PBS, the cells were incubated with $0.1 \%$ Triton X-100 (Sigma-Aldrich) for $1 \mathrm{~min}$. The cells were then washed and incubated for between $40 \mathrm{~min}$ and $1 \mathrm{~h}$ at $37^{\circ} \mathrm{C}$ in the dark with a mixture of Fast Garnet GBC, sodium nitrite, naphthol AS-BI phosphoric acid, acetate and tatrate from the Leukocyte Acid Phosphatase Assay kit (Sigma-Aldrich), according to the manufacturer's instructions. The cells were washed with distilled water, and TRAP-positive multinucleated cells containing three or more nuclei were counted under an Olympus IX71 microscope (Olympus Corporation, Tokyo, Japan).

Cell viability. The cells $\left(1 \times 10^{4}\right.$ cells/well) were seeded in a 96-well plate and incubated overnight in media supplemented with $10 \%$ FBS. Various concentrations of CGE $(5,10,20,40$ or $80 \mu \mathrm{g} / \mathrm{ml}$ ) were then added to the cells. The cells were incubated 
A

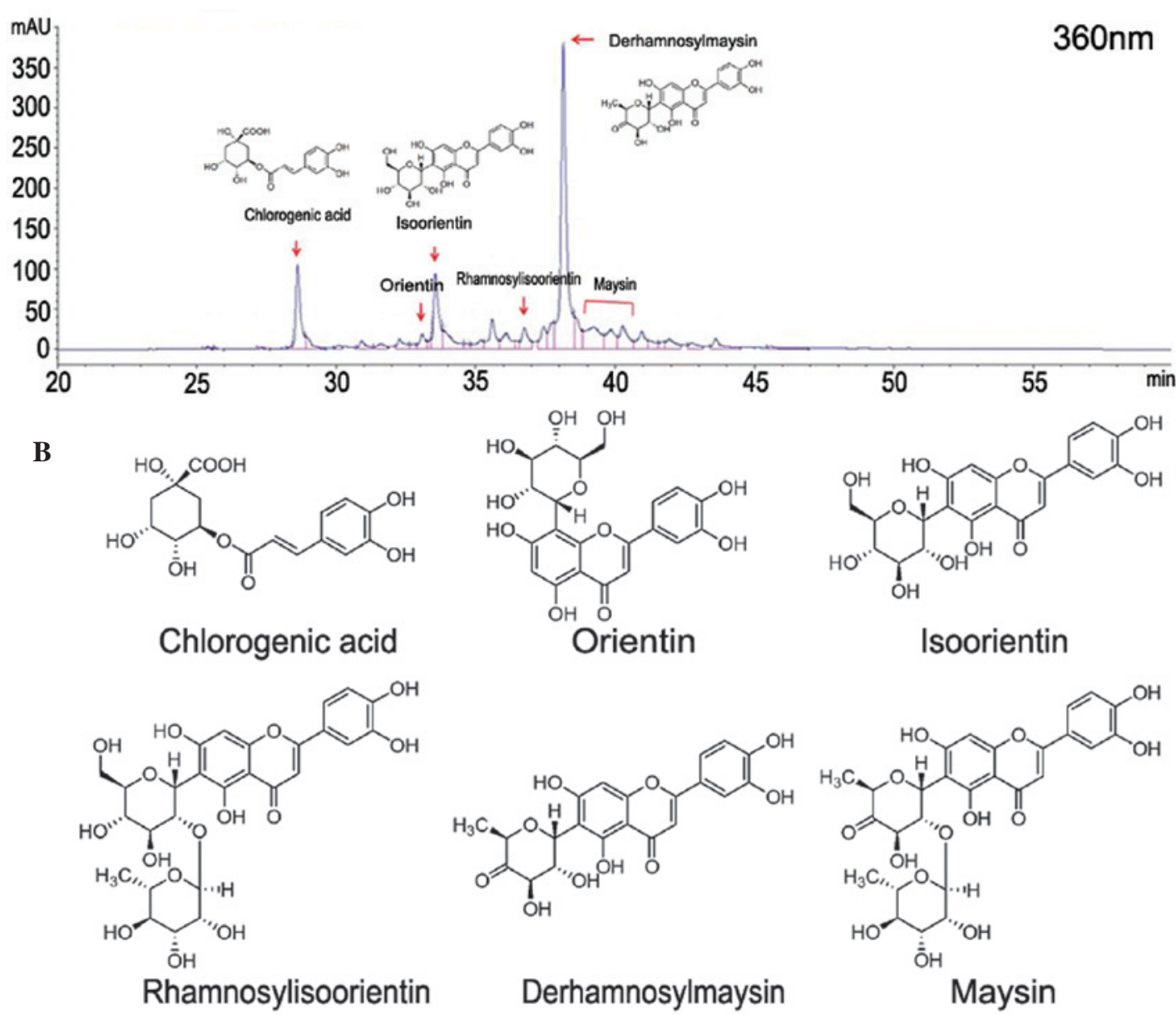

Figure 1. Composition of CGE. (A) High performance liquid chromatography chromatograph for Eremochloa ophiuroides CGEs. (B) Structures of maysin and its derivatives, isolated from Eremochloa ophiuroides CGEs. CGEs, centipedegrass extracts.

at $37^{\circ} \mathrm{C}$ in $5 \% \mathrm{CO}_{2}$ for 24 or $48 \mathrm{~h}$, washed with PBS and were then treated with medium containing $100 \mu \mathrm{g} / \mathrm{ml}$ 3-(4,5-dimethylthiazol-2-yl)-2,5-diphenyltetrazolium bromide (MTT) for $3 \mathrm{~h}$ at $37^{\circ} \mathrm{C}$. The cells were then washed with PBS and dissolved in $200 \mathrm{ml}$ DMSO. The resulting intracellular purple formazan was quantified from the absorbance at $540 \mathrm{~nm}$ using an Infinite M200 microplate reader (Tecan Group, Ltd., Männedorf, Switzerland).

Reverse transcription-polymerase chain reaction (RT-PCR). Total RNA was isolated from the cultured cells using TRIzol reagent (Molecular Research Center, Inc., Cincinnati, OH, USA) and cDNA synthesis was performed using Maxime RT Premix (iNtron Biotechnology, Inc., Seongnam, Korea), according to the manufacturer's protocol. The PCR primers were purchased from Neo Probe (Daejeon, Korea). The cDNA was amplified in a reaction mix made up to $20 \mu \mathrm{l}$, containing $1 \mu \mathrm{l}$ cDNA, Maxime PCR Premix kit (iNtron Biotechnology, Inc.), distilled water and 10 pmol of forward and of reverse primers, using the following primer sets:

c-Fos, forward 5'-CTGGTGCAGCCCACTCTGGTC-3' and reverse 5'-CTTTCAGCAGATTGGCAATCTC-3'; NFATc1, forward 5'-CAACGCCCTGACCACCGATAG-3 and reverse 5'-GGCTGCCTTCCGTCTCATAGT-3'; osteoclast-associated immunoglobulin-like receptor (OSCAR), forward 5'-CTGCTG GTAACGGATCAGCTCCCCAGA-3' and reverse 5'-CCAAGG AGCCAGAACCTTCGAAACT-3'; matrix metalloproteinase (MMP)-9, forward 5'-CGTCGTGATCCCCACTTACT-3' and reverse 5'-AGAGTACTGCTTGCCCAGGA-3'; cathepsin K, forward 5'-AGGCGGCTATATGACCACTG-3' and reverse 5'-CCGAGCCAAGAGAGCATATC-3'; dendritic cell-specific transmembrane protein (DC-STAMP), forward 5'-GCAAGG AACCCAAGGAGTCG-3' and reverse 5'-CAGTTGGCCCAG AAAGAGGG-3'; and GAPDH, forward 5'-GGTGAAGGT CGGTGTGAACG-3' and reverse 5'-CTCGCTCCTGGAAGA TGGTG-3'.

Following initial denaturation at $95^{\circ} \mathrm{C}$ for $2 \mathrm{~min}, \mathrm{PCR}$ was performed for various cycle numbers, at varying annealing temperatures, as follows: c-Fos, $58^{\circ} \mathrm{C}, 32$ cycles; NFATc1, $59^{\circ} \mathrm{C}$, 32 cycles; OSCAR, $59^{\circ} \mathrm{C}, 32$ cycles; MMP-9, $58^{\circ} \mathrm{C}, 33$ cycles; cathespin $\mathrm{K}, 59^{\circ} \mathrm{C}, 32$ cycles; and DC-STAMP, $60^{\circ} \mathrm{C}$; 31 cycles. The reaction products were separated on $1 \%$ agarose gels (Lonza Group, Basel, Switzerland) and stained with GelRed (Biotium, Hayward, CA, USA). These cycles also included denaturation steps at $95^{\circ} \mathrm{C}$ for $30 \mathrm{sec}$ and elongation steps at $72^{\circ} \mathrm{C}$ for $45 \mathrm{sec}$. The relative levels of c-Fos, NFATc1, OSCAR, MMP-9, cathepsin $\mathrm{K}$ and DC-STAMP were normalized to GAPDH. To quantify expression levels, band intensity was compared using 
Gel-Pro version 3.0 software (Exon Intron Inc., Loganville, PA, USA).

Immunoblot analysis. Following washing with cold PBS, cells were harvested and lysed using lysis buffer (Rockland, Inc., Gilbertsville, PA, USA). Following centrifugation at $18,300 \mathrm{x} \mathrm{g}$ for $20 \mathrm{~min}$ at $4^{\circ} \mathrm{C}$, the supernatants were used as cell extract. Equal concentrations of cell extract $(40 \mathrm{mg} / \mathrm{ml})$, determined using a bicinchoninic acid assay (Thermo Fisher Scientific, Inc.), were separated using SDS-PAGE on 8-12\% gels and then transferred onto polyvinylidene diflioride membranes (Bio-Rad Laboratories, Inc., Hercules, CA, USA). The membranes were blocked with $5 \%$ skim milk in Tris-buffered saline (TBS) containing 0.1\% Tween-20 (TBST) at room temperature for $1 \mathrm{~h}$, and then incubated overnight at $4^{\circ} \mathrm{C}$ with primary antibodies diluted in $5 \%$ skim milk in TBST. These were: Rabbit anti-c-Fos (dilution, 1:1,000; cat no. 2250; Cell Signaling Technology, Inc., Danvers, MA, USA); mouse anti-NFATc1 (dilution, 1:1,000; cat. no. sc-7294; Santa Cruz Biotechnology, Inc., Dallas, TX, USA); and rabbit anti-GAPDH (dilution, 1:2,000; cat. no. 2118; Cell Signaling Technology, Inc.). The membranes were washed three times in TBST for $30 \mathrm{~min}$, with shaking. The membranes were then incubated with the secondary antibodies horseradish peroxidase (HRP)-conjugated horse anti-mouse IgG (dilution, 1:2,000; cat. no. 7076; NFAT1-incubated membrane) or HRP-conjugated goat anti-rabbit IgG (dilution, 1:2,000; cat. no. 7074; all other membranes) (both Cell Signaling Technology, Inc.) for $1 \mathrm{~h}$ at room temperature. Following this, the membranes were washed twice in TBST for $10 \mathrm{~min}$, with shaking. These immunocomplexes were visualized on X-ray film (GE Healthcare Life Sciences, Chalfont, UK) using Enhanced Chemiluminescence Prime Western Blotting Detection reagent (GE Healthcare Life Sciences), according to the manufacturer's protocol. To quantify expression levels, band intensity was compared using Gel-Pro software.

Lipopolysaccharide (LPS)-induced bone resorption. A total of 21 ICR mice (6-week-old) were divided into three groups ( $n=7$ per group). The control group mice were injected twice with PBS, instead of LPS, at day 0 and day 4 (PBS group). The remaining mice received an intraperitoneal injection of LPS ( $5 \mathrm{mg} / \mathrm{kg}$ body weight) at day 0 and day 4 . Half of the LPS-treated mice were injected orally with CGE 1 day prior to LPS injection, and every day following LPS injection (LPS+CGE group). The other half of the LPS-treated mice received vehicle $(10 \mathrm{mM} \mathrm{KOH})$, instead of CGE (LPS group). All mice were sacrificed by cervical dislocation 8 days following LPS injection. The left femurs of the mice were scanned using a high-resolution micro-CT scanner (Skyscan 1076; Bruker, Kontich, Belgium). Bone trabecular parameter analyses were performed with the micro-CT data using the software provided by Skyscan. All animal experiments were performed in accordance with the principles of the Care and Use of Laboratory Animals (17), and approved by the Institutional Animal Care and Use Committee of Korea Atomic Energy Research Institute (Jeongeup Si, Korea).

Statistical analysis. All experimental data are expressed as the mean \pm standard error of the mean. All experiments were repeated at least three times, unless otherwise indicated. Statistical differences between the control and experimental groups were analyzed using Student's $t$-test with SPSS version 9.0 software (SPSS Inc., Chicago, IL, USA). P $<0.05$ was considered to indicate a statistically significant difference.

\section{Results}

CGE inhibits osteoclast differentiation. To determine the effects of CGE on osteoclastogenesis, the effect of CGE on osteoclast formation was examined using the bone marrow cells. Osteoclasts were generated from mouse BMMs in the presence of CGE in osteoclastogenesis. RANKL $(100 \mathrm{ng} / \mathrm{ml})$ induced TRAP-positive multinucleated osteoclast differentiation in the BMMs, however CGE reduced the formation and numbers of TRAP-positive multinucleated cells generated, with $76.4 \pm 10.3$, $62.7 \pm 7.9,69.5 \pm 6.8,44.6 \pm 3.0$ and $10.7 \pm 3.37 \%$ inhibition at 5,10 , 20,40 and $80 \mu \mathrm{g} / \mathrm{ml}$, respectively (Fig. 2A and B). Therefore, CGE reduced the formation and numbers of TRAP-positive multinucleated cells in a concentration-dependent manner. CGE inhibited the negative effect on osteoclastogenesis. The present study also measured the effects of CGE in the BMMs using an MTT assay to exclude the possibility that the inhibition was due to cytotoxicity. CGE demonstrated no cytotoxic effects at the concentrations found to effectively inhibit osteoclast formation (Fig. 2C). This result, suggesting that osteoclastogenesis was suppressed by CGE, did not affect cell growth rate or induce toxic effects in the BMMs.

Regulation of osteoclastogenic-specific genes in $R A N K L$-induced BMMs by CGE. Osteoclast differentiation is associated with the upregulation of specific genes in response to RANKL (7). In the present study, RANKL significantly induced the expression of MMP-9, OSCAR and cathepsin K in the BMMs. However, CGE decreased the expression of these osteoclastogenesis-associated genes in a time-dependent manner (Fig. 3). CGE did not affect the expression of the housekeeping gene, GAPDH. These data showed that CGE had a specific effect on the regulation of certain genes induced during osteoclast differentiation. This raises the possibility that CGE may inhibit osteoclast differentiation through the inhibition of the RANKL-induced expression of c-Fos and NFATc1.

CGE inhibits the expression of NFATc1 and c-Fos in RANKL-induced osteoclastogenesis. Osteoclast differentiation is regulated by the induction of various genes in response to RANKL and RANK binding. NFATc1 has been shown to be upregulated following RANKL stimulation, and is important for osteoclast differentiation (4). Therefore, the present study examined the effect of CGE on the expression levels of NFATc1 and c-Fos. The osteoclast precursors were pretreated with CGE and then stimulated with RANKL at various time points. The results revealed that the mRNA levels of c-Fos and NFATc1 were increased in response to RANKL, however the expression levels of c-Fos and NFATc1 were significantly inhibited by CGE (Fig. 4A and B). To compare with the result $s$ of the RT-PCR analyses, the present study examined the protein levels using immunobloting. The protein expression levels of NFATc1 and c-Fos 1 increased in response to RANKL, however the expression levels of c-Fos and NFATc1 decreased following 
A
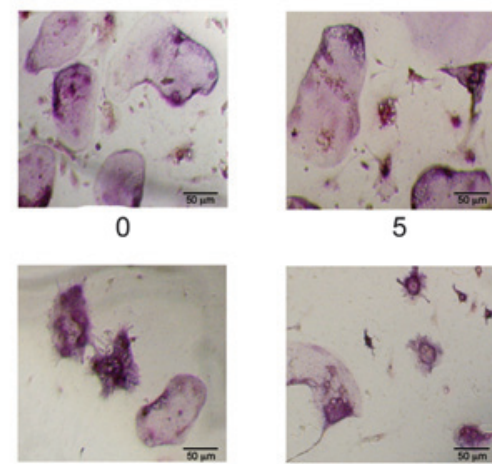

20

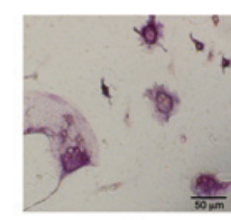

40

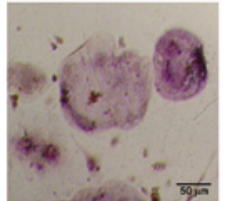

10

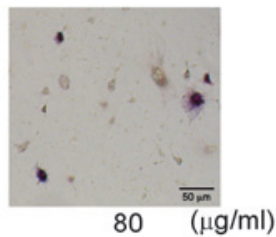

B

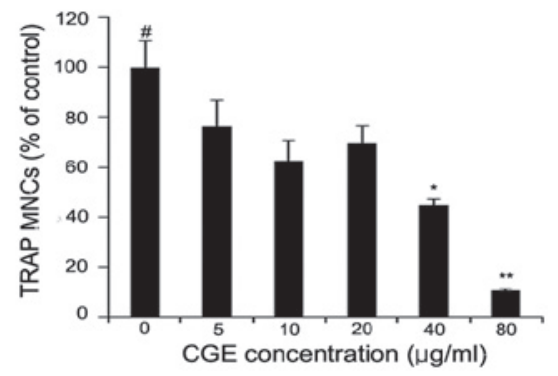

$\mathbf{C}$

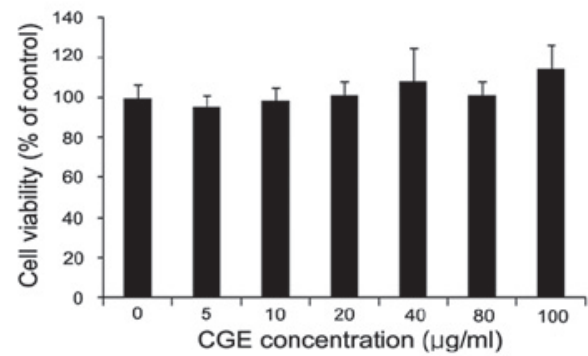

Figure 2. Effect of CGE on RANKL-mediated osteoclast differentiation. (A) BMMs were cultured for 3 days with the indicated concentrations of CGE in the presence of M-CSF (30 ng/ml) and RANKL (100 ng/ml). After 3 days, the cells were fixed and stained for TRAP. (B) TRAP-positive MNCs were counted. (C) Cytotoxicity of CGE on BMMs. The effect of CGE on cell viability was measured using a 3-(4,5-dimethylthiazol-2-yl)-2,5-diphenyltetrazolium bromide assay. The results are expressed as the mean \pm standard error of the mean. ${ }^{* *} \mathrm{P}<0.01$ and ${ }^{*} \mathrm{P}<0.05$ vs. vehicle-treated cells $\left({ }^{\#}\right)$. Magnification, $\mathrm{x} 100$. MNCs, multinucleated cells. CGEs, centipedegrass extracts; RANKL, receptor activator of nuclear factor $\kappa$-B ligand; BMMs, bone marrow-derived macrophages; M-CSF, macrophage colony-stimulating factor, TRAP, tartrate-resistant acid phosphatase.

A

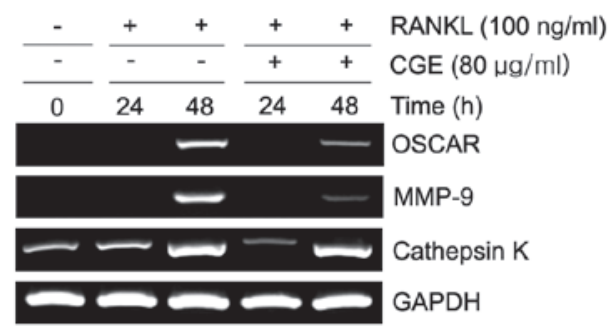

B

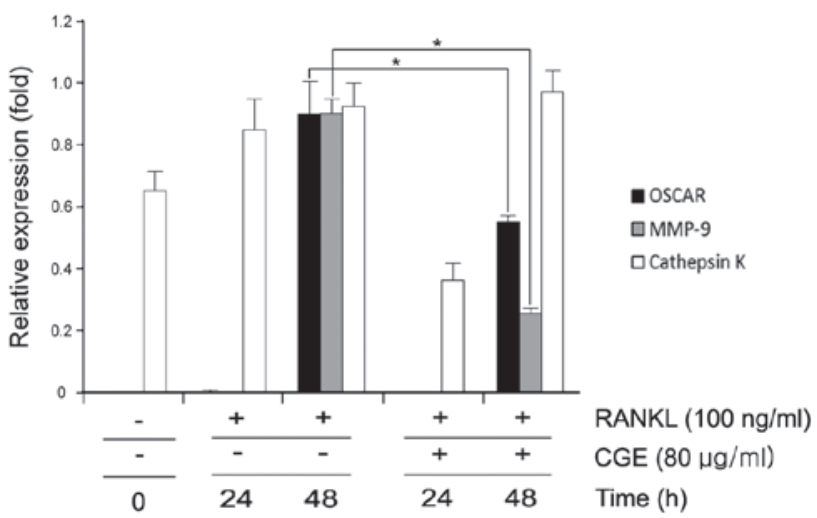

Figure 3. Suppression of RANKL-induced gene expression by CGE. (A) BMMs were pretreated with or without CGE (80 $\mu \mathrm{g} / \mathrm{ml})$ for $1 \mathrm{~h}$ prior to RANKL stimulation $(100 \mathrm{ng} / \mathrm{ml})$ at indicated time points. The mRNA expression levels of MMP-9, OSCAR and cathepsin K were determined using reverse transcription-polymerase chain reaction analysis and compared with that of GAPDH. (B) Expression from this agarose gel, relative to GAPDH. "P<0.01 vs. indicated groups. CGEs, centipedegrass extracts; RANKL, receptor activator of nuclear factor $\mathrm{\kappa}$-B ligand; BMMs, bone marrow-derived macrophages; MMP, matrix metalloproteinase; OSCAR, osteoclast-associated immunoglobulin-like receptor.

pretreatment with CGE (Fig. 4C). These results suggested that CGE inhibited oateoclastogenesis by reducing the expression levels of c-Fos and NFATc1 induced by RANKL.

CGE suppresses osteoclast cell-cell fusion through downregulation of the expression of DC-STAMP. DC-STAMP is an important regulator of osteoclast cell fusion $(15,16)$. In the present study, the number of fused TRAP-positive multinuclear osteoclasts was increased by RANKL however, CGE suppressed RANKL-induced osteoclast fusion (Fig. 2A). It order to determine the effect of CGE on the expression of DC-STAMP, RT-PCR analysis was performed. It was revealed that CGE attenuated the mRNA expression of the fusion marker, DC-STAMP, confirming the inhibitory effect of CGE on RANKL-mediated osteoclast fusion (Fig. 5A and B).

CGE on in vivo LPS-induced bone resorption. The present study investigated the effects of CGE on bone erosion in vivo. 
A
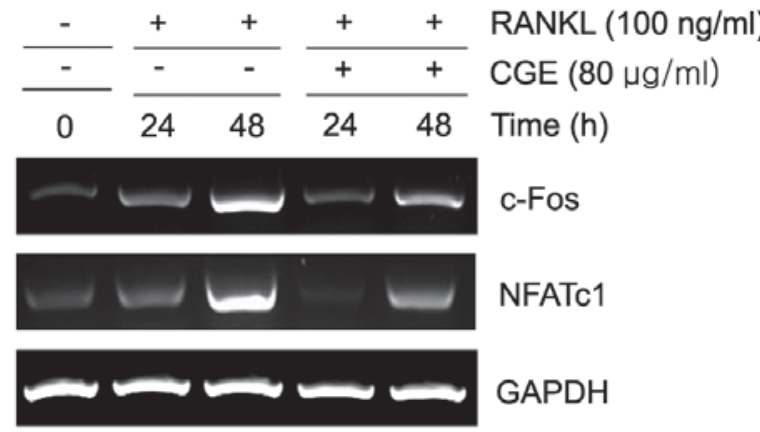

B
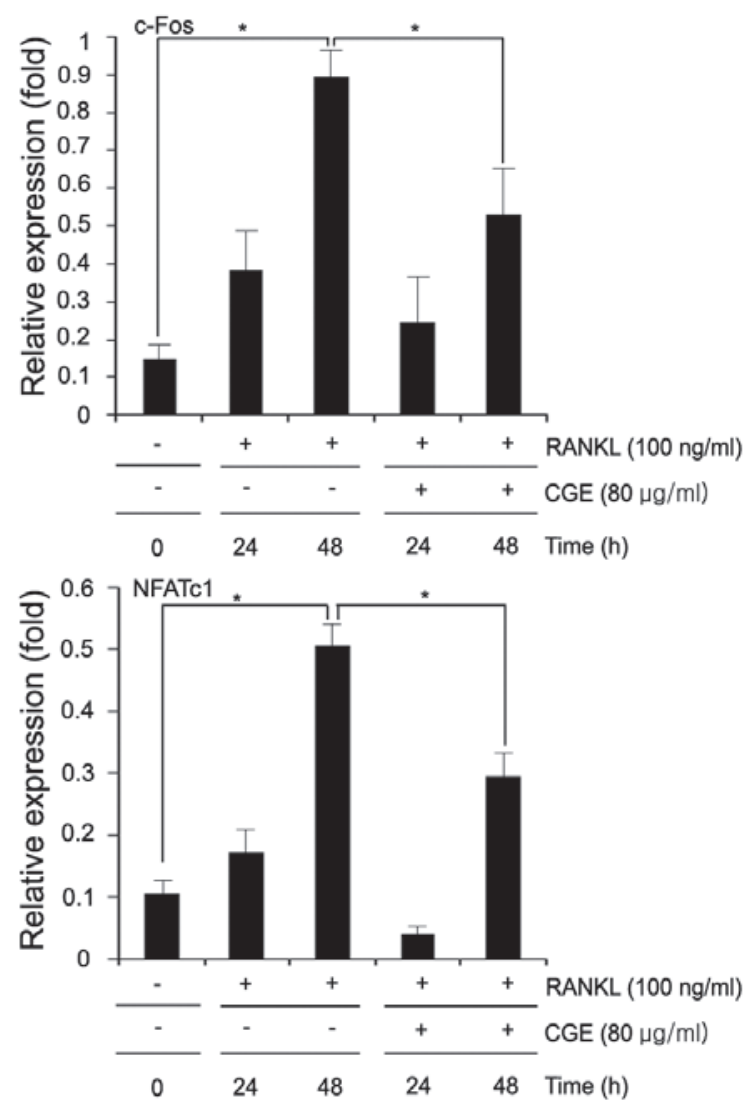

C

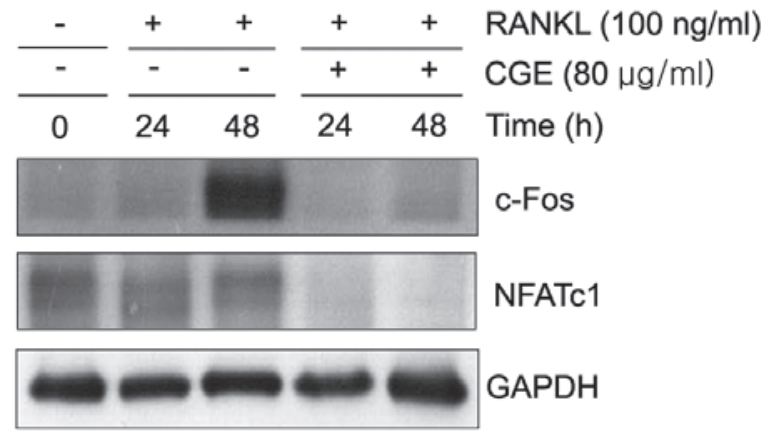

Figure 4. Inhibition of the expression levels and activities of NFATc1 and c-Fos by CGE (A and B) BMMs were pretreated with or without CGE $(80 \mu \mathrm{g} / \mathrm{ml})$ for $1 \mathrm{~h}$ prior to RANKL stimulation $(100 \mathrm{ng} / \mathrm{ml})$ at indicated time points. The mRNA expression levels of NFATc1 and c-Fos were analyzed using reverse transcription-polymerase chain reaction analysis and compared with that of GAPDH. (C) BMMs were pretreated with or without CGE $(80 \mu \mathrm{g} / \mathrm{ml})$ for $1 \mathrm{~h}$ prior to RANKL stimulation $(100 \mathrm{ng} / \mathrm{ml})$ at indicated time points. The cells were extracted using lysis buffer, and cell extracts were analyzed using immunoblot analysis with antibodies against c-Fos, NFATc1, and GAPDH. " $\mathrm{P}<0.01$ vs. indicated groups. CGEs, centipedegrass extracts; RANKL, receptor activator of nuclear factor $\kappa-B$ ligand; BMMs, bone marrow-derived macrophages; NFATc1 nuclear factor of activated T cell cytoplasmic 1 .
$\mathbf{A}$

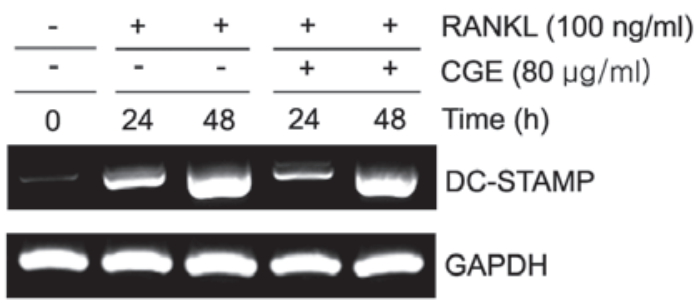

B

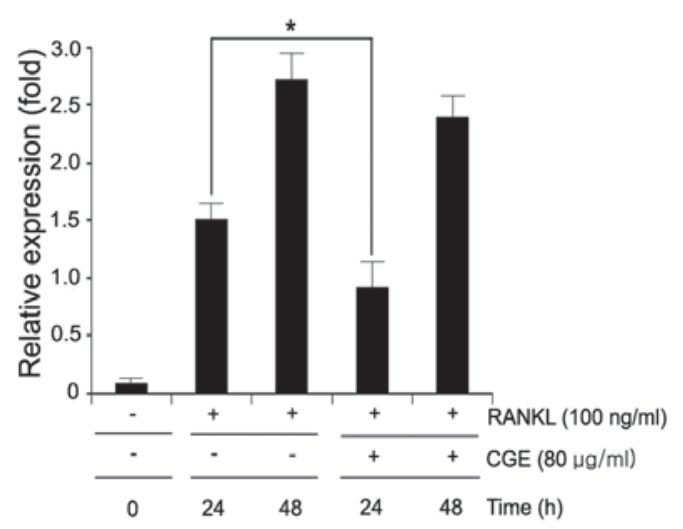

Figure 5. Regulation of the transcriptional activity of DC-STAMP. (A and B) bone marrow-derived macrophages were pretreated with or without CGE $(80 \mu \mathrm{g} / \mathrm{ml})$ for $1 \mathrm{~h}$ prior to RANKL stimulation $(100 \mathrm{ng} / \mathrm{ml})$ at indicated time points. The expression of DC-STAMP was analyzed using reverse transcription-polymerase chain reaction, and compared with that of GAPDH. ${ }^{*} \mathrm{P}<0.01$ vs. indicated groups. CGE, centipedegrass extract; RANKL, receptor activator of nuclear factor $\kappa-B$ ligand; DC-STAMP dendritic cell-specific transmembrane protein.

For this experiment, an animal model of endotoxin-induced bone resorption was used $(1,18,19)$. The mice were challenged with LPS and treated either with or without CGE. The femurs of the mice were collected 8 days following LPS injection (day 0), and the collected femurs were scanned using micro-CT. The LPS-injection mice exhibited profound decreases in trabecular and cortical bone densities of the femur. The micro-CT analyses also revealed that LPS-induced bone loss was reduced in the femurs of the CGE-treated LPS-mediated mice (Fig. 6A). To enable more detailed analyses of the effect of CGE in the bone microstructure, the femoral radiographic results were analyzed to assess the trabecular bone microstructure using measurement software. The calculation of the microstructure indices of trabecular bone density revealed the volume of trabecular bone (TB) per unit of total bone mass (TV). The BV/TV was decreased following LPS induction, and this reduction was observed, to a lesser extent, in the CGE-treated group (Fig. 6B). The LPS-induced reduction in trabecular number and bone mineral density were also attenuated, whereas trabecular separation was increased by CGE (Fig. 6B).

\section{Discussion}

Osteoclasts are dynamic cells, which are capable of resorbing bone matrix (2). The excessive formation of this cell type causes bone-destructive diseases, including osteoporosis, periodontits and rheumatoid arthritis (5).

Bone resorption is affected by various factors, which govern osteoclast number and activity (5). The major signals 
A

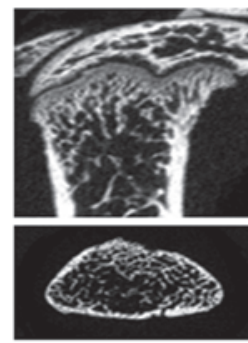

PBS

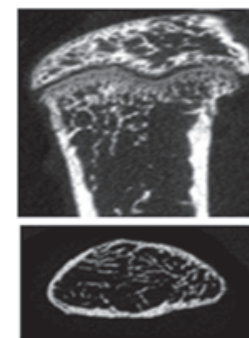

LPS
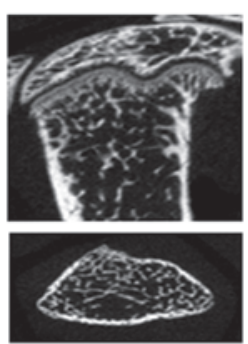

LPS + CGE

B
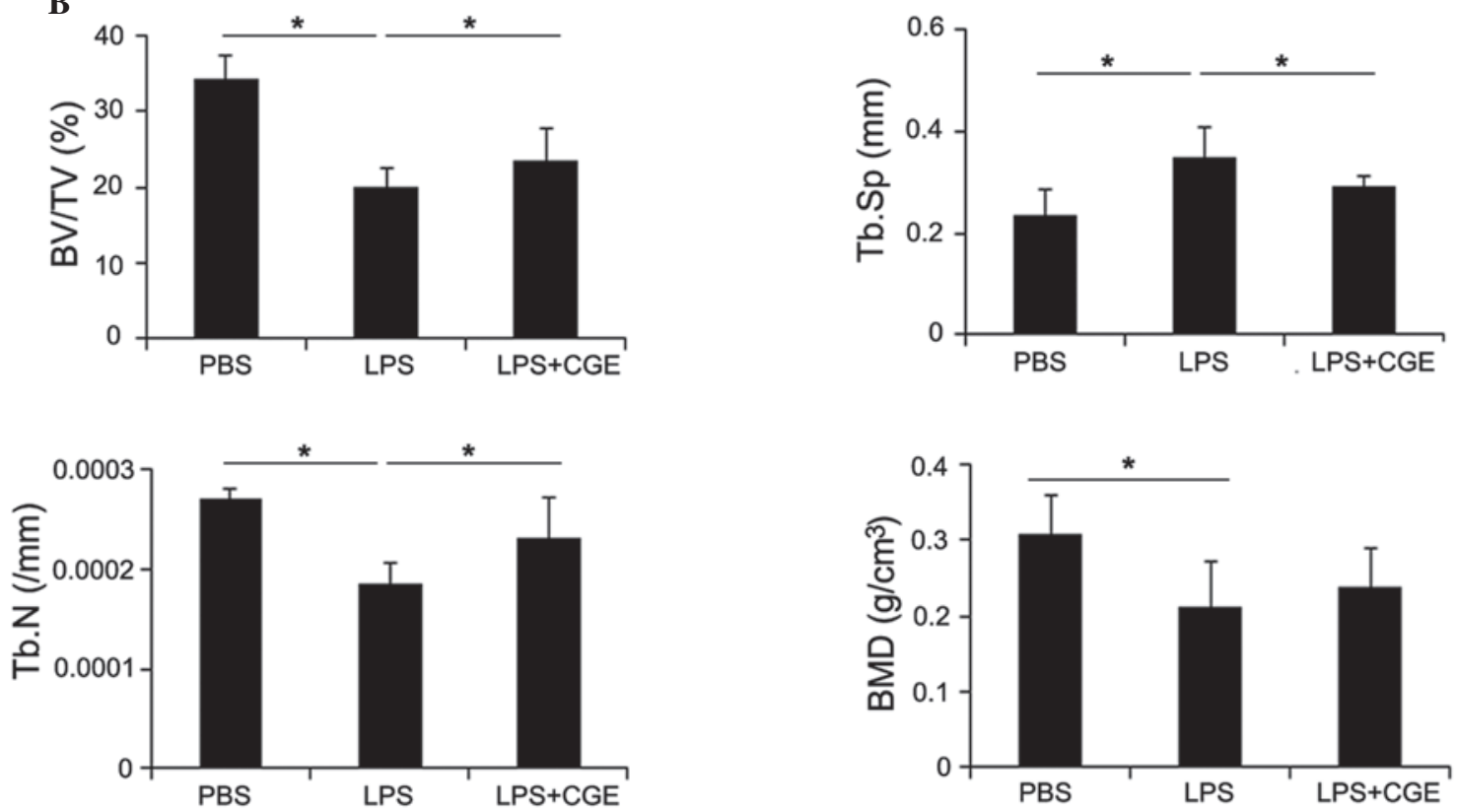

Figure 6. Inhibitory effect of CGE on LPS-induced bone resorption. ICR mice (6 weeks old) were intraperiotoneally injected twice with LPS and PBS, whereas CGE was administsered orally every day for 7 days. (A) Radiographs of longitudinal and transverse sections of the proximal femurs were obtained using a micro-CT apparatus. (B) Bone parameters was also measured using micro-CT data with Skyscan1076 micro-CT scanner software. Data are expressed as the mean \pm standard error of the mean. "P $<0.05$ vs. indicated groups. CGEs, centipedegrass extracts; PBS, phosphate-buffered saline; LPS, lipopolysaccharide; BV/TB, bone volume/trabecular volume; Tb.Sp, trabecular separation; Tb.N, trabecular number; BMD bone mineral density.

of osteoclast differentiation are RANKL and the RANK binding pathway. This binding activates TRAF6, which induces MAPKs, including ERK, JNK and p38, and also activates $\mathrm{NF}-\kappa \mathrm{B}(2)$. This signaling subsequently activates the transcription factors, c-Fos, PU.1 and NFATc1, all of which are required for osteoclastogenesis (10). These transcription factors undergo nuclear translocation and regulate the expression of several osteoclast-specific genes, including cathepsin K, TRAP, OSCAR, MMP-9 and NFATc1 (3). c-Fos causes the expression of NFATc1 during RANKL-induced osteoclast differentiation. In addition, NFATc1 can induce osteoclast differentiation in the absence of RANKL. Thus, c-Fos and NFATc1 are essential factors in osteoclast differentiation (20). Therefore, the inhibition of osteoclast formation and/or its activation may have therapeutic applications for pathological bone diseases $(2,5)$.

In the present study, it was demonstrated that CGE inhibited osteoclast generation from primary BMMs. The data suggested that CGE reduced osteoclast formation, compared to the RANKL-stimulated control groups (Fig. 2A and B), and that these results were not a result of cytotoxic effects of CGE (Fig. 2C). The interaction of RANKL and RANK results in the activation of various signaling cascades during osteoclast differentiation and activation. This signaling pathway involved three well known MAPKs, including JNK, ERK and p38, and the activation of signaling molecules induces transcription factors, including $N F-\kappa B$, NFATc1 and activator-protein-1 (AP-1), which are essential for osteoclast differentiation $(1,2,7)$. In the present study, CGE did not inhibit the activation of MAPKs or $N F-\kappa B$ in response to RANKL (data not shown). Therefore, it is unlikely that CGE directly regulated the activation of MAPKs/NF- $\kappa \mathrm{B}$ downstream signaling. The transcription factor, NFATc1, is also critical in osteoclastogenesis RANKL-RANK binding activation through intracellular TRAF6 and the c-Fos signaling pathway (1). This is a master regulator of osteoclast differentiation, which auto-amplifies and affects the expression of osteoclast-specific genes, including TRAP, calcitonin receptor, OSCAR, cathepsin K and MMP-9 (1). The present study suggested that CGE suppressed the RANKL-induced activation of c-Fos and NFATc1 (Fig. 4). Additionally, the mRNA levels of osteoclast markers, including OSCAR, MMP-9 and cathepsin K, were inhibited by CGE (Fig. 3). Multinucleated giant cells are formed by the fusion of osteoclasts, and it has been reported that DC-STAM and the $\mathrm{d} 2$ isoform of vacuolar $\left(\mathrm{H}^{+}\right)$ATPase (V-ATPase), V(0) 


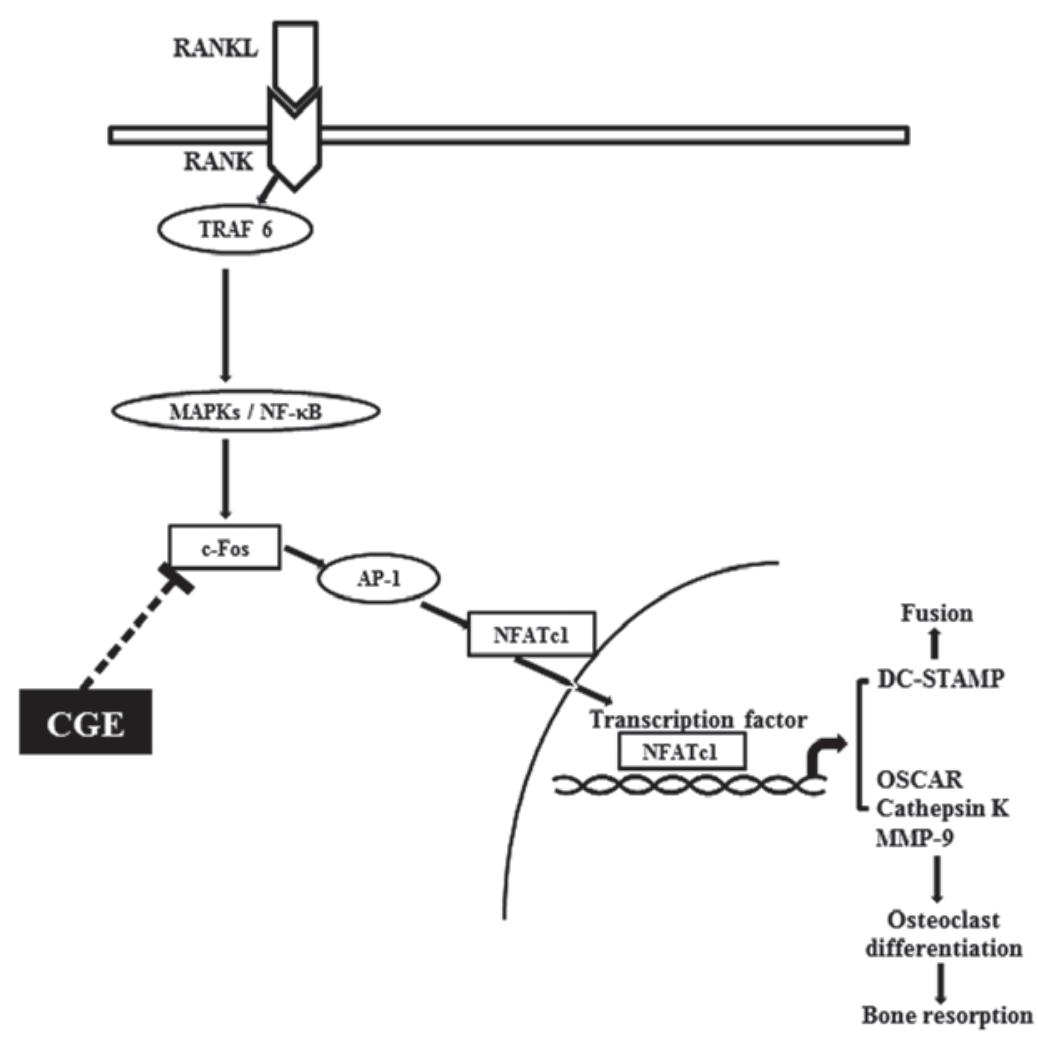

Figure 7. Schematic diagram of the inhibitory function of CGE on osteoclastogenesis in RANK-RANKL signaling. The binding of RANKL to RANK led to the activation of downstream molecules, including NF-kB and MAPK. However, these molecules were not affected by CGE. CGE reduced the induction of c-Fos and NFATc1, leading to decreased osteoclastogenesis-associated gene expression, and osteoclast formation and function. CGE, centipedegrass extract; RANKL, receptor activator of nuclear factor $\kappa-B$ ligand; MAPK, mitogen-activated protein kinase; NF- $\kappa B$, nuclear factor- $\kappa B$; TRAF6, TNF receptor-associated factor 6; AP-1, activator protein-1; NFATc1, nuclear factor of activated T cell cytoplasmic; DC-STAMP dendritic cell-specific transmembrane protein; OSCAR; osteoclast-associated immunoglobulin-like receptor; MMP, matrix metalloproteinase.

domain (Atp6v0d2), are key regulators of osteoclast cell-cell fusion, which are regulated by NFATc1 (3). The present study investigated how CGE regulates osteoclast maturation, and examined whether CGE stimulation regulated the expression of DC-STAMP $(3,21,22)$. The RANKL-induced BMMs significantly increased the expression of DC-STAMP, however, the expression of this marker was inhibited by CGE treatment, in a time-dependent manner (Fig. 5A and B). These results indicated that the osteoclast differentiation induced by RANKL required the osteoclastogenesis-associated genes regulated by NFATc1. However, CGE exposure in the BMMs suppressed the expression levels of osteoclastogenesis-associated genes, including MMP-9, OSCAR, cathepsin K and DC-STAMP, as well as c-Fos and NFATc1.

The present demonstrated the inhibitory effects of CGE on osteoclastogenesis in primary precusor cells. The present study also suggested the molecular mechanism underlying this inhibition, involving transcription factors, including NFATc1 and c-Fos, which act in genes involved in osteoclast differentiation. CGE inhibited the expression of DC-STAMP, decreasing osteoclast cell-cell fusion (Fig. 7). In addition, CGE decreased bone loss and attenuated associated parameters in the LPS-induced in vivo model (Fig. 6). The results suggested that CGE may offer potential for used in the development of a therapeutic drug for the treatment of bone-resorbing diseases, including osteoporosis, rheumatoid arthritis and advanced periodontal disease.

\section{Acknowledgements}

This study was supported by the National Research Foundation of Korea, funded by the Korean Ministry of Science, ICT and Future Planning.

\section{References}

1. Kwak HB, Lee BK, Oh J, Yeon JT, Choi SW, Cho HJ, Lee MS, Kim JJ, Bae JM, Kim SH and Kim HS: Inhibition of osteoclast differentiation and bone resorption by rotenone, through down-regulation of RANKL-induced c-Fos and NFATc1 expression. Bone 46: 724-731, 2010.

2. Kim HJ, Yoon KA, Lee MK, Kim SH, Lee IK and Kim SY: A novel small molecule, NecroX-7, inhibits osteoclast differentiation

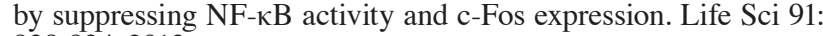
928-934, 2012.

3. Choi J, Choi SY, Lee SY, Lee JY, Kim HS, Lee SY and Lee NK: Caffeine enhances osteoclast differentiation and maturation through p38 MAP kinase/Mitf and DC-STAMP/CtsK and TRAP pathway. Cell Signal 25: 1222-1227, 2013

4. Ghayor C, Correro RM, Lange K, Karfeld-Sulzwe LS, Grätz KW and Weber FE: Inhibition of osteoclast differentiation and bone resorption by N-Methylpyrrolidone. J Biol Chem 286: 24458-24466, 2011.

5. Kim HH, Kim JH, Kwak HB, Huang H, Han SH, Ha H, Lee SW, Woo ER and Lee ZH: Inhibition of osteoclast differentiation and bone resorption by tanshinone IIA isolated from Salvia miltiorrhiza Bunge. Biochem Pharmacol 67: 1647-1656, 2004.

6. Kim BG, Kwak HB, Choi EY, Kim HS, Kim MH, Kim SH, Choi MK, Chun $\mathrm{CH}$, Oh J and Kim JJ: Amorphigenin inhibits osteoclast differentiation by suppressing c-Fos and nuclear factor of activated T cells. Anat Cell Biol 43: 310-316, 2010. 
7. Fumimoto R, Sakai E, Yamaguchi Y, Sakamoto H, Fukuma Y, Nishishita K, Okamoto K and Tsukuba T: The coffee diterpene kahweol prevents osteoclastogenesis via impairment of NFATc1 Expression and blocking of Erk phosphorylation. J Pharmacol Sci 118: 479-486, 2012.

8. Park YR, Eun JS, Choi HJ, Nepal M, Kim DK, Seo SY, Li R, Moon WS, Cho NP, Cho SD, et al: Hexane-Soluble fraction of the common fig, Ficus carica, inhibits osteoclast differentiation in murine bone marrow-derived macrophages and RAW 264.7 Cells. Korean J Physiol Pharmacol 13: 417-424, 2009.

9. Wada T, Nakashima T, Hiroshi $\mathrm{N}$ and Penninger JM: RANKL-RANK signaling in osteoclastogenesis and bone disease. Trends Mol Med 12: 17-25, 2006.

10. Mochizuki A, Takami M, Miyamoto Y, Nakamaki T, Tomoyasu S, Kadono Y, Tanaka S, Inoue T and Kamijo R: Cell adhesion signaling regulates RANK expression in osteoclast precursors. PLoS One 7: e48795, 2012.

11. Zhao Q, Wang X, Liu Y, He A and Jia R: NFATc1: Functions in osteoclasts. Int J Biochem Cell Biol 42: 576-579, 2010.

12. Badaboina S, Bai HW, Park CH, Jang DM, Choi BY and Chung BY: Molecular mechanism of apoptosis induction in skin cancer cells by the centipedegrass extract. BMC complement. Altern Med 13: 350, 2013.

13. Barampuram S, Chung BY, Lee SS, An BC, Lee EM and Cho JY: Development of an embryogenic callus induction method for centipede grass (Eremochloa ophiuroides Munro) and subsequent plant regeneration. In Vitro Cell Dev Bio Plant 45: 155-161, 2009.

14. Wiseman BR, Gueldner RC, Lynch RE and Severson RF: Biochemical activity of centipedegrass against fall armyworm larvae. J Chem Ecol 16: 2677-2690, 1990.
15. Park HJ, Chung BY, Lee MK, Song Y, Lee SS, Chu GM, Kang SN, Song YM, Kim GS and Cho JH: Centipede grass exerts anti-adipogenic activity through inhibition of C/EBP $\beta$, $\mathrm{C} / \mathrm{EBP} \alpha$, and PPAR $\gamma$ expression and the AKT signaling pathway in 3T3-L1 adipocytes. BMC Complement Altern Med 12: 230, 2012.

16. Choi HJ, Park YR, Nepal M, Choi BY, Cho NP, Choi SH, Heo SR, Kim HS, Yang MS and Soh Y: Inhibition of osteoclastogenic differentiation by Ikarisoside A in RAW 264.7 cells via JNK and NF-kappaB signaling pathways. Eur J Pharmacol 636: 28-35, 2010.

17. National Research Council (US) Committee for the Update of the Guide for the Care and Use of Laboratory Animals: Guide for the Care and Use of Laboratory Animals. 8th edition. National Academies Press (US), Washington, DC, USA, 2011.

18. Han KY, Yang D, Chang EJ, Lee Y, Huang H, Sung SH, Lee ZH, Kim YC and Kim HH: Inhibition of osteoclast differentiation and bone resorption by sauchinone. Biochem Pharmacol 74: 911-923, 2007.

19. Nepal M, Choi HJ, Choi BY, Yang MS, Chae JI, Li L and Soh Y: Hispidulin attenuates bone resorption and osteoclastogenesis via the RANKL-induced NF- $\kappa B$ and NFATc1 pathways. Eur J Pharmacol 715: 96-104, 2013.

20. Kwak HB, Yang D, Ha H, Lee JH, Kim HN, Woo ER, Lee S, $\mathrm{Kim} \mathrm{HH}$ and Lee $\mathrm{ZH}$ : Tanshinone IIA inhibits osteoclast differentiation through down-regulation of c-Fos and NFATc1. Exp Mol Med 38: 256-264, 2006.

21. Yagi M, Miyamoto T, Sawatani Y, Iwamoto K, Hosogane N, Fujita N, Morita K, Ninomiya K, Suzuki T, Miyamoto K, et al: DC-STAMP is essential for cell-cell fusion in osteoclasts and foreign body giant cells. J Exp Med 202: 345-351, 2005.

22. Yagi M, Miyamoto T, Toyama Y and Suda T: Role of DC-STAMP in cellular fusion of osteoclasts and macrophage giant cells. J Bone Miner Metab 24: 355-358, 2006 